\title{
Drenaje de absceso retroperitoneal por lumboscopia: Presentación de un caso
}

\author{
Castillo O,***, Vitagliano G*, Díaz M*, Hoyos J*, Pinto I*, C*, Estrada JC*. \\ "Unidad de Laparoscopia Urológica, Clínica Santa María. ${ }^{* *}$ Departamento de Urología, Facultad de \\ Medicina, Universidad de Chile. Santiago de Chile
}

Actas Urol Esp. 2006;30(7):711-713

\section{RESUMEN \\ DRENAJE DE ABSCESO RETROPERITONEAL POR VIA LUMBOSCOPICA: PRESENTACION \\ DE UN CASO}

Las publicaciones existentes evalúan el manejo de las necrosis pancreáticas infectadas. Dichas son tratadas por diversos autores en forma percutánea o por la vía retroperitoneoscópica. La morbilidad y mortalidad de dicha patología prevalece por encima de la vía empleada para su resolución dificultando la evaluación objetiva del método empleado. Presentamos un caso de drenaje de una colección pancreática por retroperitoneoscopia con resolución favorable evidenciando la escasa morbilidad y las ventajas de este método

Palabras clave: Pancreatitis. Absceso retroperitoneal. Laparoscopia.

\section{ABSTRACT}

LUMBOSCOPIC DRAINAGE OF RETROPERITONEAL ABSCESS. CASE REPORT

Existing reports evaluate the management of infected pancreatic necrosis. Most of the authors choose between percutaneous and retroperitoneoscopic drainage. The high morbidity and mortality of this pathology distorts the objective evaluation of the treatment employed. With present a case of infected pancreatic collection favorably resolved with retroperitoneoscopic drainage evidencing the low morbidity of this technique.

Keywords: Pancreatic. Retroperitoneal abscess. Laparoscopy.

$\mathrm{U}$ na de las complicaciones más temidas de la pancreatitis y asociada a un peor pronóstico es el desarrollo de necrosis pancreática infectada. Esta se presenta en un bajo porcentaje de los casos y requiere de un enérgico tratamiento clínico y quirúrgico. A su vez también pueden existir colecciones pancreáticas que pueden infectarse sin presentar un cuadro tan devastador como el de las primeras. Para éstas es necesario realizar un eficiente drenaje de la colección acompañado del tratamiento antibiótico adecuado. El drenaje puede ser en forma abierta, percutánea o mediante retroperitoneoscópica.
Presentamos nuestra experiencia de una colección pancreática infectada de resolución favorable por vía retroperitoneoscópica.

\section{CASO CLINICO}

Paciente masculino de 56 años de edad con antecedentes de serologia positiva para HIV y Hepatitis B que presenta cuadro de colecistitis aguda por la cual se le realiza colecistectomia laparoscopica de urgencia.

Durante el post-operatorio tardío, al mes de la cirugia, el paciente presenta un bilioma el cual es evacuado en forma percutánea. A su vez también 
se realiza una derivación biliar mediante colangiografia endoscopica.

Producto de la anterior el paciente presenta una pancreatitis aguda asociándose a ésta una colección peri-pancreática. Dicha colección se evacua mediante un drenaje percutáneo inicial, el cual prueba ser insuficiente presentando el paciente una importante colección retroperitoneal. Sin presentar mejoría, y con un débito persistente por el drenaje asociado a fiebre y deterioro del estado general, se realiza tomografia computada de abdomen y pelvis. Ésta informa líquido encapsulado con nivel hidroaéreo en el flanco derecho adyacente a la pared abdominal extendiéndose $17 \mathrm{~cm}$ en el sentido cráneo-caudal y 11 x $3 \mathrm{~cm}$ en diámetro transverso (Figs. 1 y 2).

Con diagnostico de absceso parietocólico derecho extraperitoneal se realizo drenaje mediante

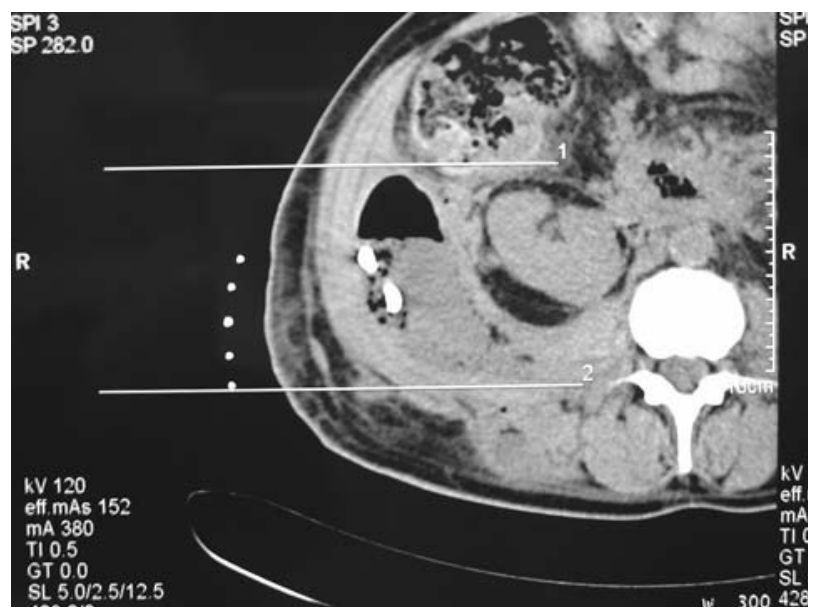

FIGURA 1

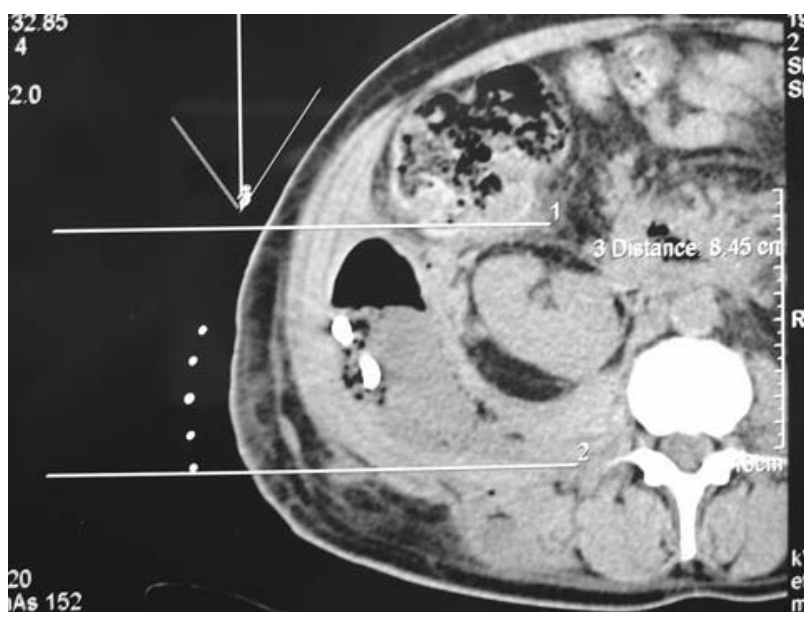

FIGURA 2 lumboscopia, evacuando abundante material necrotico y purulento.

Se colocaron dos drenajes de silicona pura enfrentados, confeccionando un sistema de lavado continuo, utilizando solución fisiológica a goteo lento (Figs. 3 y 4).

El paciente evolucionó en forma favorable con escasos registros febriles. Presentó un cultivo positivo para Pseudomona Aureaginosa, la cual fue tratada con Meropenem durante la hospitalización. Al cuarto día postoperatorio se reemplazó el lavado continuo por un sistema de hemosuctor y fue dado de alta.

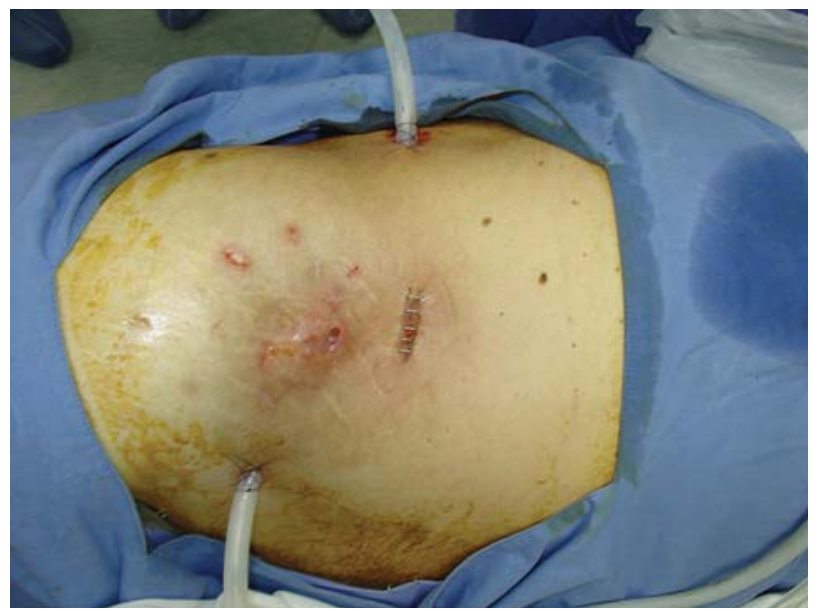

FIGURA 3

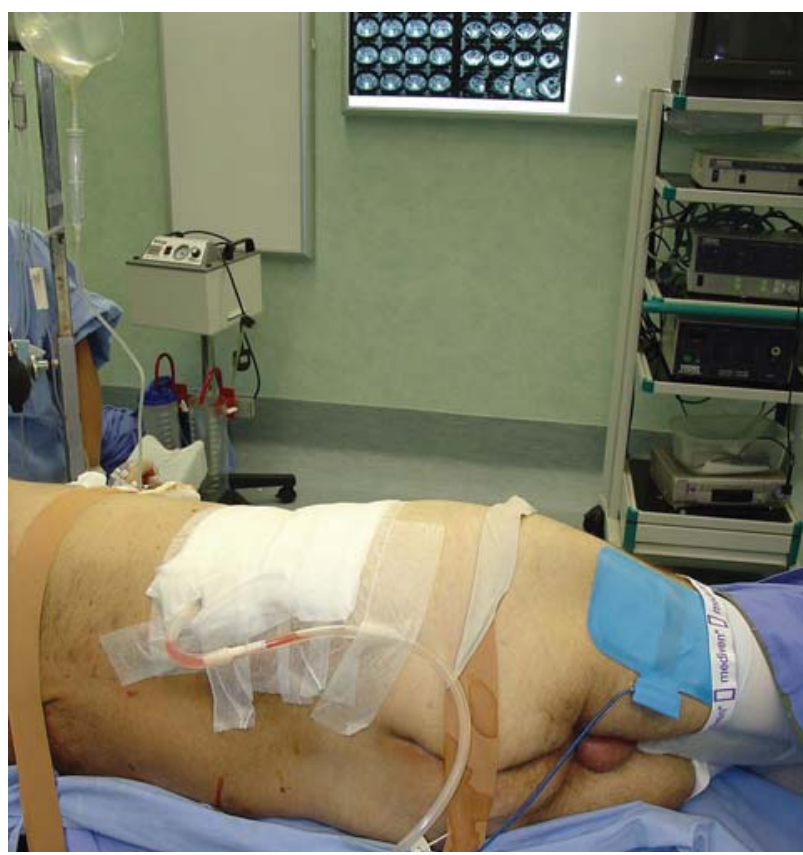

FIGURA 4 
Durante los controles, el paciente continuó afebril retirándose el drenaje tras cesar el debito del mismo.

\section{DISCUSION}

Existen escasa bibliografía acerca del manejo retroperitoneoscópico de colecciones pancreáticas infectadas. La gran mayoría de las publicaciones hacen referencia al drenaje y manejo de las colecciones en los casos de necrosis pancreáticas infectadas en pacientes con gran compromiso sistémico. La cirugía a cielo abierto fue la primera en ser utilizada para esta patología. Entre 1976 y 1988 se encuentran muchas series publicadas utilizando este tipo de abordaje. Con el advenimiento de nuevas tecnologías y la búsqueda de métodos cada vez menos invasivos, el drenaje a cielo abierto fue reservado para casos particulares. Muchos autores prefieren la vía percutánea para el drenaje de dichas colecciones.

Otros reportes hacen referencia a la realización de necrosectomías en forma laparoscópica. Castellanos ${ }^{3}$ en una serie de 15 pacientes realizada en el Hospital Universitario Virgen de Arrixaca en Murcia, España reporta un 27\% de mortalidad y un $20 \%$ de morbilidad utilizando la retroperitoneoscopia para el drenaje de necrosis pancreáticas infectadas. Queda evidenciado que la morbilidad y mortalidad de la patología pancreática prevalece sobre el método utilizado para su resolución. Esto dificulta la evaluación objetiva del método empleado.

A diferencia de la mayoría de las series, el paciente reportado no presentaba una pancreatitis necrótica severa, sino una colección infectada como complicación de su pancreatitis. Dicho cuadro no comprometía la vida del paciente pero enlentecía su recuperación, agregando morbilidad. Se utilizó la laparoscopia por vía retroperitoneal utilizando tres trócares de los cuales dos fueron de $10 \mathrm{~mm}$. Para asegurar el éxito del procedimiento se dejó un lavado continuo con solución fisiológica utilizando los puertos de $10 \mathrm{~mm}$. El cuadro fue resuelto en forma satisfactoria mediante la retroperitoneoscopia y el paciente no presentó complicaciones asociadas a dicha técnica. Concluimos que el drenaje de colecciones retroperitoneales por vía laparoscópica es un método de baja morbilidad que posibilita la utilización de lavado continuo y permite un eventual control endoscópico, de ser éste necesario.

\section{REFERENCIAS}

1. Van Vyve EL, Reynaert MS, Lengele BG, Pringot JT, Otte JB, Kestens PJ. Retroperitoneal-laparostomy: A surgical treatment of pancreatic abscesses after an acute necrotizing pancreatitis. Surgery. 1992;111(4):369-375.

2. Cheung MT, Ho CN, Siu KW, Kwok PC. Percutaneous Drainage and Necrosectomy in the management of pancreatic necrosis. ANZ J Surg. 2005;75(4):204-7.

3. Castellanos, Gregorio MD, PhD; Pinero, Antonio MD, PhD; Serrano, Andres Md; Parrilla, Pascual MD, PhD. Infected Pancreatic Necrosis: Translumbar Approach and Management with Retroperitoneoscopy. Arch Surg. 2002 Sep; 137(9): 1060-1063.

Dr. O. Castillo

E-mail: octaviocastillo@vtr.net

(Trabajo recibido el 8 de septiembre 2006) 